\title{
A decomposition of the universal embedding space for the near polygon $\mathbb{H}_{n}$
}

\author{
Bart De Bruyn \\ Ghent University, Department of Mathematics, Krijgslaan 281 (S22), B-9000 \\ Gent, Belgium, E-mail: bdb@cage.ugent.be
}

\begin{abstract}
Let $\mathbb{H}_{n}, n \geq 1$, be the near $2 n$-gon defined on the 1 -factors of the complete graph on $2 n+2$ vertices, and let $e$ denote the absolutely universal embedding of $\mathbb{H}_{n}$ into $\mathrm{PG}(W)$, where $W$ is a $\frac{1}{n+2}\left(\begin{array}{c}2 n+2 \\ n+1\end{array}\right)$ dimensional vector space over the field $\mathbb{F}_{2}$ with two elements. For every point $z$ of $\mathbb{H}_{n}$ and every $i \in \mathbb{N}$, let $\Delta_{i}(z)$ denote the set of points of $\mathbb{H}_{n}$ at distance $i$ from $z$. We show that for every pair $\{x, y\}$ of mutually opposite points of $\mathbb{H}_{n}, W$ can be written as a direct sum $W_{0} \oplus W_{1} \oplus \cdots \oplus W_{n}$ such that the following four properties hold for every $i \in\{0, \ldots, n\}$ : (1) $\left\langle e\left(\Delta_{i}(x) \cap \Delta_{n-i}(y)\right)\right\rangle=\mathrm{PG}\left(W_{i}\right) ;(2)$ $\left\langle e\left(\bigcup_{j \leq i} \Delta_{j}(x)\right)\right\rangle=\mathrm{PG}\left(W_{0} \oplus W_{1} \oplus \cdots \oplus W_{i}\right) ;(3)\left\langle e\left(\bigcup_{j \leq i} \Delta_{j}(y)\right)\right\rangle=$ $\operatorname{PG}\left(W_{n-i} \oplus W_{n-i+1} \oplus \cdots \oplus W_{n}\right) ;(4) \operatorname{dim}\left(W_{i}\right)=\left|\Delta_{i}(x) \cap \Delta_{n-i}(y)\right|=$ $\left(\begin{array}{c}n \\ i\end{array}\right)^{2}-\left(\begin{array}{c}n \\ i-1\end{array}\right) \cdot\left(\begin{array}{c}n \\ i+1\end{array}\right)$.
\end{abstract}

Keywords: projective embedding, generating set, universal embedding

MSC2000: 51A45, 15A75, 51A 50

\section{Introduction}

Let $\mathbb{H}_{n}, n \geq 1$, be the following point-line geometry:

- The points of $\mathbb{H}_{n}$ are the partitions of $\{1,2, \ldots, 2 n+2\}$ in $n+1$ subsets of size 2 .

- The lines of $\mathbb{H}_{n}$ are the partitions of $\{1,2, \ldots, 2 n+2\}$ in $n-1$ subsets of size 2 and 1 subset of size 4 .

- A point is incident with a line if and only if the partition corresponding to the point is a refinement of the partition corresponding to the line. 
The point-line geometry $\mathbb{H}_{n}, n \geq 1$, is a so-called dense near polygon with three points per line. An alternative description of $\mathbb{H}_{n}$ can be given where the points are the 1 -factors of a complete graph on $2 n+2$ vertices. Indeed, there exists a natural bijective correspondence between the partitions of $\{1,2, \ldots, 2 n+2\}$ in $n+1$ subsets of size 2 and the 1 -factors of the complete graph with vertex set $\{1,2, \ldots, 2 n+2\}$.

The near polygon $\mathbb{H}_{n}, n \geq 1$, is embeddable into a projective space and hence admits the so-called absolutely universal embedding.

For every two points $x$ and $y$ of $\mathbb{H}_{n}$ (i.e. partitions $x$ and $y$ of $\{1, \ldots, 2 n+$ $2\}$ in $n+1$ subsets of size 2), let $\Gamma_{x, y}$ denote the graph with vertex set $\{1,2, \ldots, 2 n+2\}$ and edge set $x \cup y$. Then the distance $\mathrm{d}(x, y)$ between $x$ and $y$ in the collinearity graph of $\mathbb{H}_{n}$ is equal to $n+1-N$, where $N$ denotes the number of connected components of $\Gamma_{x, y}$. If $x$ is a point of $\mathbb{H}_{n}$ and $i \in \mathbb{N}$, then $\Delta_{i}(x)$ denotes the set of points at distance $i$ from $x$ and $\Delta_{i}^{*}(x)$ the set of points at distance at most $i$ from $x$.

A set $S$ of points of $\mathbb{H}_{n}$ is called a subspace if every line of $\mathbb{H}_{n}$ which has at least two points in $S$ has all its points in $S$. If the smallest subspace of $\mathbb{H}_{n}$ which contains a given set $X$ of points coincides with the whole set of points of $\mathbb{H}_{n}$, then $X$ is called a generating set of $\mathbb{H}_{n}$. In Blokhuis and Brouwer [1], it was mentioned that if $x$ and $y$ are two opposite points of $\mathbb{H}_{n}$ and if $C(x, y)$ denotes the union of all geodesics from $x$ to $y$, then $C(x, y)$ is a generating set of $\mathbb{H}_{n}$ whose size is equal to the Catalan number $\frac{1}{n+2}\left(\begin{array}{c}2 n+2 \\ n+1\end{array}\right)$. In the present paper, we refine this result in the following way.

Theorem 1.1 Let $x$ and $y$ be two opposite points of the near polygon $\mathbb{H}_{n}$, $n \geq 1$, and put $X_{i}:=\Delta_{i}(x) \cap \Delta_{n-i}(y), i \in\{0, \ldots, n\}$. Then

(1) $\left|X_{i}\right|=\left(\begin{array}{c}n \\ i\end{array}\right)^{2}-\left(\begin{array}{c}n \\ i-1\end{array}\right) \cdot\left(\begin{array}{c}n \\ i+1\end{array}\right)$;

(2) $X_{0} \cup X_{1} \cup \cdots \cup X_{n}$ is a generating set of $\mathbb{H}_{n}$.

In the previous theorem and elsewhere in the paper, we have adopted the convention that $\left(\begin{array}{l}n \\ j\end{array}\right)=0$ for every $n \in \mathbb{N}$ and every $j \in \mathbb{Z} \backslash\{0, \ldots, n\}$. Using Theorem 1.1, we are able to prove a decomposition theorem for the absolutely universal embedding of $\mathbb{H}_{n}$.

Theorem 1.2 Let e denote the absolutely universal embedding of $\mathbb{H}_{n}, n \geq 1$, into $\mathrm{PG}(W)$, where $W$ is a $\frac{1}{n+2}\left(\begin{array}{c}2 n+2 \\ n+1\end{array}\right)$-dimensional vector space over the field $\mathbb{F}_{2}$ with two elements. Then for every pair $\{x, y\}$ of mutually opposite points of $\mathbb{H}_{n}, W$ can be written as a direct sum $W_{0} \oplus W_{1} \oplus \cdots \oplus W_{n}$ such that the following four properties hold for every $i \in\{0, \ldots, n\}$ :

(1) $\left\langle e\left(\Delta_{i}(x) \cap \Delta_{n-i}(y)\right)\right\rangle=\mathrm{PG}\left(W_{i}\right)$;

(2) $\left\langle e\left(\Delta_{i}^{*}(x)\right)\right\rangle=\mathrm{PG}\left(W_{0} \oplus W_{1} \oplus \cdots \oplus W_{i}\right)$; 
(3) $\left\langle e\left(\Delta_{i}^{*}(y)\right)\right\rangle=\mathrm{PG}\left(W_{n-i} \oplus W_{n-i+1} \oplus \cdots \oplus W_{n}\right)$;

(4) $\operatorname{dim}\left(W_{i}\right)=\left(\begin{array}{c}n \\ i\end{array}\right)^{2}-\left(\begin{array}{c}n \\ i-1\end{array}\right) \cdot\left(\begin{array}{c}n \\ i+1\end{array}\right)$.

Remark A. In the literature, decomposition theorems for other projective embeddings of dense near polygons have been proved:

- the Grassmann embeddings of symplectic dual polar spaces [5, Theorem 1.1], see also [8] for another approach;

- the spin-embeddings of some dual polar spaces and the near polygons $\mathbb{H}_{n}$ and $\mathbb{I}_{n}[6$, Theorem 1.7]; $1.4]$.

- the Grassmann embeddings of Hermitian dual polar spaces [7, Theorem

Remark B. Theorems 1.1 and 1.2 can also be deduced from the decomposition theorem for the Grassmann embedding of the symplectic dual polar space $D W(2 n-1,2)$. Such an approach would be highly artificial and not very elegant. Indeed, a proof of Theorem 1.1 which needs the introduction of a $2 n$-dimensional vector space $V$ equipped with a nondegenerate alternating bilinear form and technical computations in the exterior algebra of $V$ is quite a detour. The approach discussed in the present paper avoids all this machinery. Notice that in the proof of Theorem 1.2, we introduce a symplectic dual polar space $D W(2 n-1,2)$ but we only need to invoke some elementary properties of this dual polar space.

\section{Preliminaries}

\subsection{A recursively defined series of numbers}

In this section, we define in a recursive way numbers $f_{n}(k, l), n \in \mathbb{N} \backslash\{0,1\}$ and $k, l \in\{0, \ldots, n\}$, and give a closed expression for these numbers.

The numbers $f_{2}(k, l), k, l \in\{0,1,2\}$, are defined in the following table.

\begin{tabular}{|c||c|c|c|}
\hline$f_{2}(k, l)$ & $l=0$ & $l=1$ & $l=2$ \\
\hline \hline$k=0$ & 1 & 1 & 0 \\
\hline$k=1$ & 0 & 1 & 1 \\
\hline$k=2$ & 0 & 1 & 0 \\
\hline
\end{tabular}

For every $n \geq 3$ and $k, l \in\{0, \ldots, n\}$, we define

- If $k$ is even and $l=n$, then we define $f_{n}(k, l):=0$.

- If $k$ is odd and $l=0$, then we define $f_{n}(k, l):=0$. 
- If $k=0$ and $l \neq n$, then we define $f_{n}(k, l):=\sum_{i=0}^{n-1} f_{n-1}(i, l)$.

- If $k \neq 0$ is even and $l \neq n$, then we define $f_{n}(k, l):=\sum_{i=k-1}^{n-1} f_{n-1}(i, l)$.

- If $k$ is odd and $l \neq 0$, then we define $f_{n}(k, l):=\sum_{i=k-1}^{n-1} f_{n-1}(i, l-1)$.

It was show in De Bruyn [5, Section 2] that for every $n \in \mathbb{N} \backslash\{0,1\}$ and all $k, l \in\{0,1, \ldots, n\}$, we have

$f_{n}(k, l)=\left(\begin{array}{c}n-1-\left\lfloor\frac{k}{2}\right\rfloor \\ l-\left\lfloor\frac{k+1}{2}\right\rfloor\end{array}\right) \cdot\left(\begin{array}{c}n-\left\lfloor\frac{k+1}{2}\right\rfloor \\ l+\frac{(-1)^{k}-1}{2}\end{array}\right)-\left(\begin{array}{c}n-1-\left\lfloor\frac{k}{2}\right\rfloor \\ l-1-\left\lfloor\frac{k+1}{2}\right\rfloor\end{array}\right) \cdot\left(\begin{array}{c}n-\left\lfloor\frac{k+1}{2}\right\rfloor \\ l+\frac{(-1)^{k}+1}{2}\end{array}\right)$.

\subsection{The big maxes of $\mathbb{H}_{n}$}

A near polygon is a partial linear space $\mathcal{S}=(\mathcal{P}, \mathcal{L}, \mathrm{I}), \mathrm{I} \subseteq \mathcal{P} \times \mathcal{L}$, with the property that for every point $x$ and every line $L$, there exists a unique point on $L$ nearest to $x$. Here, distances are measured in the collinearity graph $\Gamma$ of $\mathcal{S}$. If $n$ is the diameter of $\Gamma$, then the near polygon is called a near $2 n$-gon. A near 0-gon is just a point and a near 2-gon is a line. Near quadrangles are usually called generalized quadrangles (Payne and Thas [10]).

A near polygon is called dense if every line is incident with at least three points and if every two points at distance 2 from each other have at least two common neighbors. By Theorem 4 of Brouwer and Wilbrink [3], every two points of a dense near $2 n$-gon at distance $\delta \in\{0, \ldots, n\}$ from each other are contained in a unique convex sub- $2 \delta$-gon. These sub- $2 \delta$-gons are called quads if $\delta=2$ and maxes if $\delta=n-1$. The existence of quads in a dense near polygon was already shown by Shult and Yanushka [12, Proposition 2.5].

A $\max M$ of a dense near polygon $\mathcal{S}$ is called big in $\mathcal{S}$ if every point $x$ of $\mathcal{S}$ not contained in $M$ is collinear with a necessarily unique point $\pi_{M}(x)$ of $M$. If $M$ is big in $\mathcal{S}$ and $x$ is a point of $\mathcal{S}$ not contained in $M$, then $\mathrm{d}(x, y)=1+\mathrm{d}\left(\pi_{M}(x), y\right)$ for every point $y$ of $M$. If $M$ is big in $\mathcal{S}$ and every line of $\mathcal{S}$ is incident with precisely three points, then a reflection $\mathcal{R}_{M}$ about $M$ can be defined which is an automorphism of $\mathcal{S}$ (see [4, Theorem 1.11]). If $x \in M$, then we define $\mathcal{R}_{M}(x):=x$. If $x \notin M$, then $\mathcal{R}_{M}(x)$ denotes the unique point of the line $x \pi_{M}(x)$ different from $x$ and $\pi_{M}(x)$. More information on dense near polygons can be found in the book [4].

Let $\mathbb{H}_{n}, n \geq 2$, be the dense near $2 n$-gon defined on the partitions of $\{1,2, \ldots, 2 n+2\}$ in $n+1$ subsets of size 2 (see Section 1 ). There exists a bijective correspondence between the big maxes of $\mathbb{H}_{n}$ and the subsets of size 2 of $\{1,2, \ldots, 2 n+2\}$. If $\{i, j\}$ is a subset of size 2 of $\{1,2, \ldots, 2 n+2\}$, then the set of all partitions $P$ of $\{1,2, \ldots, 2 n+2\}$ for which $\{i, j\} \in P$ is a big 
$\max M[i, j]$ of $\mathbb{H}_{n}$. Conversely, every big $\max$ of $\mathbb{H}_{n}$ is obtained in this way. The point-line geometry $\widetilde{M}$ induced on a big $\max M$ (by the points and lines which are contained in it) is isomorphic to $\mathbb{H}_{n-1}$. Suppose now that $\left\{i_{1}, j_{1}\right\}$ and $\left\{i_{2}, j_{2}\right\}$ are two distinct subsets of size 2 of $\{1,2, \ldots, 2 n+2\}$. If $\left\{i_{1}, j_{1}\right\} \cap\left\{i_{2}, j_{2}\right\}=\emptyset$, then the big maxes $M\left[i_{1}, j_{1}\right]$ and $M\left[i_{2}, j_{2}\right]$ meet. If $\left\{i_{1}, j_{1}\right\} \cap\left\{i_{2}, j_{2}\right\}$ is a singleton, say $\left\{i_{1}\right\}=\left\{i_{2}\right\}$, then the reflection of $M\left[i_{1}, j_{1}\right]$ about $M\left[i_{2}, j_{2}\right]=M\left[i_{1}, j_{2}\right]$ is equal to the big $\max M\left[j_{1}, j_{2}\right]$. More information about the near polygon $\mathbb{H}_{n}$ can be found in [4, Section 6.2].

\subsection{The absolutely universal embedding of $\mathbb{H}_{n}$}

By Ronan [11], every point-line geometry $\mathcal{S}=(\mathcal{P}, \mathcal{L}, \mathrm{I}), \mathrm{I} \subseteq \mathcal{P} \times \mathcal{L}$ with three points per line which is fully embeddable into a projective space admits the absolutely universal embedding which is obtained in the following way. Let $V$ be a vector space over the field $\mathbb{F}_{2}$ with a basis $B$ whose vectors are indexed by the elements of $\mathcal{P}$, say $B=\left\{\bar{e}_{p} \mid p \in \mathcal{P}\right\}$. Let $W$ denote the subspace of $V$ generated by all vectors $\bar{e}_{p_{1}}+\bar{e}_{p_{2}}+\bar{e}_{p_{3}}$, where $\left\{p_{1}, p_{2}, p_{3}\right\}$ is a line of $\mathcal{S}$. Then the map $p \in \mathcal{P} \mapsto\left\{\bar{e}_{p}+W, W\right\}$ defines a full embedding of $\mathcal{S}$ into the projective space $\operatorname{PG}(V / W)$. This full embedding is isomorphic to the so-called absolutely universal embedding of $\mathcal{S}$.

The absolutely universal embedding of the near polygon $\mathbb{H}_{n}, n \geq 1$, is described in Blokhuis and Brouwer [1, Section 3]. Let $V$ be a $(2 n+2)$ dimensional vector space over $\mathbb{F}_{2}$ with basis $\left\{\bar{e}_{1}, \bar{e}_{2}, \ldots, \bar{e}_{2 n+2}\right\}$. For every point $P=\left\{\left\{i_{1}, i_{2}\right\},\left\{i_{3}, i_{4}\right\}, \ldots,\left\{i_{2 n+1}, i_{2 n+2}\right\}\right\}$ of $\mathbb{H}_{n}$, put $e(P)$ equal to the point $\left\langle\left(\bar{e}_{i_{1}}+\bar{e}_{i_{2}}\right) \wedge\left(\bar{e}_{i_{3}}+\bar{e}_{i_{4}}\right) \wedge \cdots \wedge\left(\bar{e}_{i_{2 n+1}}+\bar{e}_{i_{2 n+2}}\right)\right\rangle$ of $\mathrm{PG}\left(\bigwedge^{n+1} V\right)$. Then $e$ defines a full embedding of $\mathbb{H}_{n}$ into a subspace of $\mathrm{PG}\left(\bigwedge^{n+1} V\right)$ of dimension $\frac{1}{n+2}\left(\begin{array}{c}2 n+2 \\ n+1\end{array}\right)$. This projective embedding is isomorphic to the absolutely universal embedding of $\mathbb{H}_{n}$.

\section{Proof of Theorem 1.1}

\subsection{A generating set of points of $\mathbb{H}_{n}$}

Suppose the points of $\mathbb{H}_{n}, n \geq 1$, are the 1-factors of the complete graph $K_{2 n+2}$, and suppose the $2 n+2$ vertices of $K_{2 n+2}$ are drawn as the vertices of a convex $(2 n+2)$-gon $\mathbb{P}$ in the plane. Blokhuis and Brouwer [1] proved that the set $Y^{*}$ of all 1-factors of $K_{2 n+2}$ without crossing edges is a generating set of $\mathbb{H}_{n}$. They also mentioned that the cardinality of $Y^{*}$ is equal to the Catalan number $\frac{1}{n+2}\left(\begin{array}{c}2 n+2 \\ n+1\end{array}\right)$, and referred to van Lint [9, Section 3.1] for a proof of this fact. 
In [1] another more geometric description of the generating set $Y^{*}$ was given. Suppose $x$ and $y$ are two opposite vertices of the near polygon $\mathbb{H}_{n}$ such that $x$ and $y$ are alternating edges of $\mathbb{P}$. Then the above generating set $Y^{*}$ of vertices coincides with the union $C(x, y)$ of all geodesics between $x$ and $y$. The proof given in [1] seems however not to be valid. (The claims $c(x \cup z)+c(y \cup z)=n+1-\operatorname{cr}(z)$ and $\mathrm{d}(x, z)+\mathrm{d}(y, z)=n-1+\operatorname{cr}(z)$ on lines $-15,-14$ and -13 of page 300 do have counter examples; in fact, there might exist 1-factors $z$ for which $c r(z)>n+1$.) We will now give a proof of this claim since we will need it in the present paper.

Proposition 3.1 The generating set $Y^{*}$ is equal to $C(x, y)$.

Proof. We start with the proof of two similar properties.

Claim I. If $z \in C(x, y)$, then there exists an edge in $z$ which is also an edge of $x$ or $y$.

Proof. Since $\mathrm{d}(x, z)+\mathrm{d}(z, y)=n$, we have $\mathrm{d}(x, z) \leq \frac{n}{2}$ or $\mathrm{d}(y, z) \leq \frac{n}{2}$.

Suppose $k:=\mathrm{d}(x, z) \leq \frac{n}{2}$ and let $x=z_{0}, z_{1}, \ldots, z_{k}=z$ be a shortest path between $x$ and $z$. The 1 -factor $x$ has $n+1$ edges. Let $N_{i}, i \in\{0, \ldots, k\}$, denote the number of edges of $x$ which are also edges of $z_{i}$. Then $N_{0}=n+1$ and $\left|N_{i}-N_{i+1}\right| \leq 2$ for every $i \in\{0, \ldots, k-1\}$. Hence, $N_{k} \geq N_{0}-2 k \geq$ $(n+1)-2 \cdot \frac{n}{2}=1$. So, there is an edge in $z$ which is also an edge of $x$.

In a similar way, one proves that if $\mathrm{d}(y, z) \leq \frac{n}{2}$, then there is an edge in $z$ which is also an edge of $y$. (qed)

Claim II. If $z \in Y^{*}$, then there is an edge of $z$ which is also an edge of either $x$ or $y$.

Proof. We define a distance function $\operatorname{dist}(\cdot, \cdot)$ on the set of vertices of $\mathbb{P}$. If $i_{1}$ and $i_{2}$ are two vertices of $\mathbb{P}$, then $\operatorname{dist}\left(i_{1}, i_{2}\right)$ is the smallest nonnegative integer $k$ for which there exist $k+1$ vertices $j_{0}, j_{1}, \ldots, j_{k}$ of $\mathbb{P}$ satisfying (a) $j_{0}=i_{1}$, (b) $j_{k}=i_{2}$, (c) $j_{i-1}, j_{i}$ are neighboring vertices of $\mathbb{P}$ for every $i \in\{1, \ldots, k\}$.

Now, let $\left\{i_{1}, i_{2}\right\}$ be an edge of $z$ for which $\operatorname{dist}\left(i_{1}, i_{2}\right)$ is as small as possible and suppose that $\operatorname{dist}\left(i_{1}, i_{2}\right)>1$. Let $i_{3}$ be a vertex of $\mathbb{P}$ which lies on a shortest path $\gamma$ from $i_{1}$ to $i_{2}$ and let $i_{4}$ be the unique vertex of $\mathbb{P}$ such that $\left\{i_{3}, i_{4}\right\}$ is an edge of $z$. Since there are no crossing edges of $z$, also $i_{4}$ is contained on the path $\gamma$. It follows that $\operatorname{dist}\left(i_{3}, i_{4}\right)<\operatorname{dist}\left(i_{1}, i_{2}\right)$, contradicting the minimality of $\operatorname{dist}\left(i_{1}, i_{2}\right)$. Hence, $\operatorname{dist}\left(i_{1}, i_{2}\right)=1$ and the edge $\left\{i_{1}, i_{2}\right\}$ of $z$ is also an edge of either $x$ or $y$. (qed)

We will now prove the proposition by induction on $n \geq 1$. Suppose first that $n=1$. Label the vertices of $\mathbb{P}$ with the numbers $1,2,3$ and 4 such that $x=\{\{1,2\},\{3,4\}\}$ and $y=\{\{2,3\},\{1,4\}\}$. One has $C(x, y)=Y^{*}=\{x, y\}$. 
We will now suppose that $n \geq 2$. By Claims I and II, it suffices to prove that $z \in Y^{*} \Leftrightarrow z \in C(x, y)$ for 1 -factors $z$ which contain a given edge $\left\{i_{1}, i_{2}\right\}$ of either $x$ or $y$. Without loss of generality, we may suppose that $\left\{i_{1}, i_{2}\right\}$ is an edge of $x$. Let $i_{0}, i_{3}$ denote the unique vertex of $\mathbb{P}$ such that $\left\{i_{0}, i_{1}\right\}$ and $\left\{i_{2}, i_{3}\right\}$ are edges of $Y$. Let $K_{2 n}$ denote the complete graph on the set of vertices of $\mathbb{P}$ distinct from $i_{1}$ and $i_{2}$ and let $\mathbb{H}_{n-1}$ denote the near polygon defined on the 1-factors of $K_{2 n}$. Let $x^{\prime}$ denote the 1-factor of $K_{2 n}$ obtained from $x$ by removing the edge $\left\{i_{1}, i_{2}\right\}$ and let $y^{\prime}$ denote the 1 -factor of $K_{2 n}$ obtained from $y$ by removing the edges $\left\{i_{0}, i_{1}\right\},\left\{i_{2}, i_{3}\right\}$ and adding the edge $\left\{i_{0}, i_{3}\right\}$. Then $x^{\prime}$ and $y^{\prime}$ are opposite vertices of $\mathbb{H}_{n-1}$. For every 1 -factor $w$ of $K_{2 n}$, let $\theta(w)$ denote the 1-factor of $K_{2 n+2}$ obtained from $w$ by adding the edge $\left\{i_{1}, i_{2}\right\}$. Then $\theta$ defines an isomorphism between $\mathbb{H}_{n-1}$ and a big max $M$ of $\mathbb{H}_{n}$. We have $\theta\left(x^{\prime}\right)=x$ and $\theta\left(y^{\prime}\right)$ is the unique point of $M$ collinear with $y$. Moreover, $\mathrm{d}(y, u)=1+\mathrm{d}\left(\theta\left(y^{\prime}\right), u\right)$ for every point $u$ of $M$. The following should now be obvious:

(a) A point $u$ of $\mathbb{H}_{n-1}$ lies on a shortest path between $x^{\prime}$ and $y^{\prime}$ if and only if $\theta\left(u^{\prime}\right)$ lies on a shortest path between $x$ and $y$.

(b) By the induction hypothesis, a point $u$ of $\mathbb{H}_{n-1}$ lies on a shortest path between $x^{\prime}$ and $y^{\prime}$ if and only if $u$, regarded as 1 -factor of $K_{2 n}$, has no crossing edges.

(c) $u$, regarded as a 1-factor of $K_{2 n}$ has no crossing edges if and only if the 1-factor $\theta(u)$ of $K_{2 n+2}$ has no crossing edges.

By (a), (b), (c) above, the statement $z \in Y^{*} \Leftrightarrow z \in C(x, y)$ holds for all 1factors $z$ which contain the edge $\left\{i_{1}, i_{2}\right\}$. This was precisely what we needed to prove.

As mentioned above, Blokhuis and Brouwer [1] proved that the set $Y^{*}$ is a generating set of $\mathbb{H}_{n}$. In view of Proposition 3.1, it is then clear that also the set $C(x, y)$ is a generating set of points of $\mathbb{H}_{n}$. This fact can also be shown in a direct way.

Proposition $3.2 C(x, y)$ is a generating set of $\mathbb{H}_{n}$.

Proof. We will prove the proposition by induction on $n$. Obviously, the proposition holds if $n=1$. So, we will suppose that $n \geq 2$. We will regard the points of $\mathbb{H}_{n}$ as partitions of $\{1,2, \ldots, 2 n+2\}$ in $n+1$ subsets of size 2 . Without loss of generality, we may suppose that $x=\{\{1,2\},\{3,4\}, \ldots,\{2 n+$ $1,2 n+2\}\}$ and $y=\{\{2,3\},\{4,5\}, \ldots,\{2 n+2,1\}\}$. Let $S$ denote the smallest subspace of $\mathbb{H}_{n}$ containing $C(x, y)$.

We will prove that all big maxes $M[i, i+1], i \in\{1, \ldots, 2 n+1\}$, are contained in $S$. If $i$ is odd, then $x \in M[i, i+1]$ and $y \notin M[i, i+1]$. In 
this case, we define $x^{\prime}:=x$ and $y^{\prime}$ denotes the unique point of $M[i, i+1]$ collinear with $y$. If $i$ is even, then $x \notin M[i, i+1]$ and $y \in M[i, i+1]$. In this case, we define $y^{\prime}:=y$ and $x^{\prime}$ denotes the unique point of $M[i, i+1]$ collinear with $x$. Then $C\left(x^{\prime}, y^{\prime}\right) \subseteq C(x, y)$. Since $x^{\prime}$ and $y^{\prime}$ are opposite points of $\widehat{M[i, i+1]} \cong \mathbb{H}_{n-1}$, the smallest subspace of $\mathbb{H}_{n}$ containing $C\left(x^{\prime}, y^{\prime}\right)$ coincides with $M[i, i+1]$ by the induction hypothesis. Hence, $M[i, i+1] \subseteq S$.

Notice that if $i_{1}, i_{2}$ and $i_{3}$ are three distinct elements of $\{1,2, \ldots, 2 n+2\}$ such that $M\left[i_{1}, i_{2}\right] \subseteq S$ and $M\left[i_{1}, i_{3}\right] \subseteq S$, then also $M\left[i_{2}, i_{3}\right] \subseteq S$ since $M\left[i_{2}, i_{3}\right]$ is the reflection of $M\left[i_{1}, i_{3}\right]$ about $M\left[i_{1}, i_{2}\right]$. By the previous paragraph it then follows that all big maxes $M[i, j], i, j \in\{1, \ldots, 2 n+2\}$ and $i \neq j$, are contained in $S$. Since every point of $\mathbb{H}_{n}$ is contained in a big max, $C(x, y)$ is a generating set of $\mathbb{H}_{n}$.

The following proposition improves Claim I of Proposition 3.1.

Proposition 3.3 Let $z \in C(x, y)$ and let $E$ denote a set of $n+1$ consecutive edges of the polygon $\mathbb{P}$. Then there is an edge in $z$ which is contained in $E$.

Proof. We label the points of $\mathbb{P}$ by the numbers $1,2, \ldots, 2 n+2$, either clockwise or counterclockwise. Without loss of generality, we may suppose that $E=\{\{1,2\},\{2,3\}, \ldots,\{n+1, n+2\}\}$. Let $\left\{j, i_{j}\right\}$ denote the edge of $z$ containing the vertex with label $j \in\{1, \ldots, n+1\}$. If $i_{j}=j+1$ for a certain $j \in\{1, \ldots, n+1\}$, then we are done.

In the sequel, we suppose that $i_{j} \neq j+1$ for every $j \in\{1, \ldots, n+1\}$ and derive a contradiction. We prove by induction on $j \in\{1, \ldots, n+1\}$ that $(a)$ $i_{j}>j+1$ and (b) $i_{j}<i_{j-1}$ if $j \neq 1$. Since $i_{1} \neq 2$, these claims hold if $j=1$. So, suppose $j \in\{2, \ldots, n+1\}$. Since $\left\{j-1, i_{j-1}\right\}$ and $\left\{j, i_{j}\right\}$ are non-crossing edges and $i_{j-1}>j$, we have that $i_{j} \in\left\{j+1, \ldots, i_{j-1}-1\right\}$. Since $i_{j} \neq j+1$, we have $i_{j}>j+1$ and $i_{j}<i_{j-1}$.

In particular, we have $i_{n+1} \leq i_{1}-n \leq 2 n+2-n=n+2$ and $i_{n+1}>n+2$, clearly a contradiction.

\subsection{The sizes of the sets $\Delta_{i}(x) \cap \Delta_{n-i}(y)$}

Consider the near polygon $\mathbb{H}_{n}, n \geq 2$, whose points are the partitions of $\{1,2, \ldots, 2 n+2\}$ in $n+1$ subsets of size 2 . Let $x$ and $y$ be two points of $\mathbb{H}_{n}$ at maximal distance from each other. Since the automorphism group of $\mathbb{H}_{n}$ acts transitively on the ordered pairs of opposite points of $\mathbb{H}_{n}$, we may without loss of generality suppose that $x=\{\{1,2\},\{3,4\}, \cdots,\{2 n+1,2 n+2\}\}$ and $y=$ $\{\{2,3\},\{4,5\}, \ldots,\{2 n, 2 n+1\},\{2 n+2,1\}\}$. For every $i \in\{1,2, \ldots, 2 n+1\}$, we define $M_{i}:=M[i, i+1]$. We also define $M_{2 n+2}:=M[1,2 n+2]$. We call $\left\{M_{1}, M_{2}, \ldots, M_{2 n+2}\right\}$ the nice set of big maxes of $\mathbb{H}_{n}$ induced by $(x, y)$. 
The following corollary is an immediate consequence of Proposition 3.3.

Corollary 3.4 We have $C(x, y) \subseteq M_{1} \cup M_{2} \cup \cdots \cup M_{n+1}$.

For all $k, l \in\{0, \ldots, n\}$, let $Y_{n}(k, l)$ denote the set of all points of $\Delta_{l}(x) \cap$ $\Delta_{n-l}(y)$ which are contained in $M_{k+1} \backslash\left(M_{1} \cup \cdots \cup M_{k}\right)$ and put $g_{n}(k, l):=$ $\left|Y_{n}(k, l)\right|$. Notice that the sets $Y_{n}(k, l), k, l \in\{0, \ldots, n\}$, are mutually disjoint. Notice also that since $M_{k} \cap M_{k+1}=\emptyset$, we also have $M_{k+1} \backslash\left(M_{1} \cup\right.$ $\left.\cdots \cup M_{k}\right)=M_{k+1} \backslash\left(M_{1} \cup \cdots \cup M_{k-1}\right)$. (Here, $M_{1} \cup \cdots \cup M_{k}=\emptyset$ if $k=0$ and $M_{1} \cup \cdots \cup M_{k-1}=\emptyset$ if $k \in\{0,1\}$.) The following is an immediate consequence of Corollary 3.4.

Corollary 3.5 (1) For every $l \in\{0, \ldots, n\}$, we have $\Delta_{l}(x) \cap \Delta_{n-l}(y)=$ $\bigcup_{0 \leq k \leq n} Y_{n}(k, l)$.

(2) $C(x, y)=\bigcup_{0 \leq k, l \leq n} Y_{n}(k, l)$.

Lemma 3.6 We have $g_{2}(0,0)=1, g_{2}(0,1)=1, g_{2}(0,2)=0, g_{2}(1,0)=0$, $g_{2}(1,1)=1, g_{2}(1,2)=1, g_{2}(2,0)=0, g_{2}(2,1)=1$ and $g_{2}(2,2)=0$.

Proof. We have $x=\{\{1,2\},\{3,4\},\{5,6\}\}, y=\{\{2,3\},\{4,5\},\{1,6\}\}$, $M_{1}=M[1,2], M_{2}=M[2,3]$ and $M_{3}=M[3,4]$. It is straightforward to verify that

$$
\begin{gathered}
Y_{2}(0,0)=\{\{\{1,2\},\{3,4\},\{5,6\}\}\}=\{x\}, \\
Y_{2}(0,1)=\{\{\{1,2\},\{4,5\},\{3,6\}\}\}, \\
Y_{2}(1,1)=\{\{\{2,3\},\{5,6\},\{1,4\}\}\}, \\
Y_{2}(1,2)=\{\{\{2,3\},\{4,5\},\{1,6\}\}\}=\{y\}, \\
Y_{2}(2,1)=\{\{\{3,4\},\{1,6\},\{2,5\}\}\}, \\
Y_{2}(0,2)=Y_{2}(1,0)=Y_{2}(2,0)=Y_{2}(2,2)=\emptyset .
\end{gathered}
$$

The values of $g_{2}(k, l), k, l \in\{0,1,2\}$, follow.

In Lemmas 3.7, 3.8, 3.9 and 3.10 below, we suppose that $n \geq 3$ and that $k$ and $l$ are elements of the set $\{0, \ldots, n\}$.

Lemma 3.7 (1) If $k$ is even and $l=n$, then $g_{n}(k, l)=0$.

(2) If $k$ is odd and $l=0$, then $g_{n}(k, l)=0$.

Proof. (1) If $k$ is even, then the $\max M_{k+1}$ does not contain $y$. Hence, $g_{n}(k, l)=0$ if $k$ is even and $l=n$.

(2) If $k$ is odd, then the $\max M_{k+1}$ does not contain $x$. Hence, $g_{n}(k, l)=0$ if $k$ is odd and $l=0$. 
Lemma 3.8 If $k=0$ and $l \neq n$, then $g_{n}(k, l)=\sum_{i=0}^{n-1} g_{n-1}(i, l)$.

Proof. The $\max M_{1}$ contains the point $x$, but not the point $y$. Let $y^{\prime}$ denote the unique point of $M_{1}$ collinear with $y$. Then $x$ and $y^{\prime}$ are opposite points of $\widetilde{M}_{1}$ and $\Delta_{l}(x) \cap \Delta_{n-l}(y) \cap M_{1}=\Delta_{l}(x) \cap \Delta_{n-1-l}\left(y^{\prime}\right) \cap M_{1}$. By Corollary $3.5(1)$, the size of $\Delta_{l}(x) \cap \Delta_{n-1-l}\left(y^{\prime}\right) \cap M_{1}$ is equal to $\sum_{i=0}^{n-1} g_{n-1}(i, l)$. This equals the size $g_{n}(0, l)$ of $\Delta_{l}(x) \cap \Delta_{n-l}(y) \cap M_{1}$.

Lemma 3.9 If $k \neq 0$ is even and $l \neq n$, then $g_{n}(k, l)=\sum_{i=k-1}^{n-1} g_{n-1}(i, l)$.

Proof. The $\max M_{k+1}$ contains the point $x$, but not the point $y$. Let $y^{\prime}$ denote the unique point of $M_{k+1}$ collinear with $y$. Then $x$ and $y^{\prime}$ are opposite points of $\widetilde{M_{k+1}}$ and $\Delta_{l}(x) \cap \Delta_{n-l}(y) \cap M_{k+1}=\Delta_{l}(x) \cap \Delta_{n-1-l}\left(y^{\prime}\right) \cap$ $M_{k+1}$. Suppose the points of the near polygon $\mathbb{H}_{n-1}$ are the partitions of $\{1,2, \ldots, 2 n+2\} \backslash\{k+1, k+2\}$ in $n$ subsets of size 2 . For every point $u$ of $\mathbb{H}_{n-1}$, let $\theta(u)$ denote the partition of $\{1,2, \ldots, 2 n+2\}$ obtained from $u$ by adding the subset $\{k+1, k+2\}$. Then $\theta$ defines an isomorphism between $\mathbb{H}_{n-1}$ and $\widetilde{M_{k+1}}$. We have

$$
\begin{gathered}
\theta^{-1}(x)=\{\{1,2\}, \ldots,\{k-1, k\},\{k+3, k+4\}, \ldots,\{2 n+1,2 n+2\}\}, \\
\theta^{-1}\left(y^{\prime}\right)=\{\{2,3\}, \ldots,\{k-2, k-1\},\{k, k+3\},\{k+4, k+5\}, \ldots,\{2 n+2,1\}\} .
\end{gathered}
$$

Now, define the following maxes:

$$
\begin{aligned}
M_{i}^{\prime} & :=M[i, i+1] \cap M_{k+1}, \quad i \in\{1, \ldots, k-1\} ; \\
M_{k}^{\prime} & :=M[k, k+3] \cap M_{k+1} ; \\
M_{i}^{\prime} & :=M[i+2, i+3] \cap M_{k+1}, \quad i \in\{k+1, \ldots, 2 n-1\} ; \\
M_{2 n}^{\prime} & :=M[1,2 n+2] \cap M_{k+1} .
\end{aligned}
$$

Then it is clear that $\left\{M_{1}^{\prime}, M_{2}^{\prime}, \ldots, M_{2 n}^{\prime}\right\}$ is the nice set of maxes of $\widetilde{M_{k+1}} \cong$ $\mathbb{H}_{n-1}$ induced by $\left(x, y^{\prime}\right)$. The $g_{n}(k, l)$ points of $\Delta_{l}(x) \cap \Delta_{n-l}(y) \cap M_{k+1}$ which are not contained in $M_{1} \cup M_{2} \cup \cdots \cup M_{k}$ are precisely the points of $\Delta_{l}(x) \cap$ $\Delta_{n-1-l}\left(y^{\prime}\right) \cap M_{k+1}$, which are not contained in $M_{1}^{\prime} \cup M_{2}^{\prime} \cup \cdots \cup M_{k-1}^{\prime}$. By Corollary $3.5(1)$, there are $\sum_{i=k-1}^{n-1} g_{n-1}(i, l)$ such points.

Lemma 3.10 If $k$ is odd and $l \neq 0$, then $g_{n}(k, l)=\sum_{i=k-1}^{n-1} g_{n-1}(i, l-1)$.

Proof. The $\max M_{k+1}$ contains the point $y$, but not the point $x$. Let $x^{\prime}$ denote the unique point of $M_{k+1}$ collinear with $x$. Then $x^{\prime}$ and $y$ are opposite points of $\widetilde{M_{k+1}}$ and $\Delta_{l}(x) \cap \Delta_{n-l}(y) \cap M_{k+1}=\Delta_{l-1}\left(x^{\prime}\right) \cap \Delta_{n-l}(y) \cap$ $M_{k+1}$. Suppose the points of the near polygon $\mathbb{H}_{n-1}$ are the partitions of $\{1,2, \ldots, 2 n+2\} \backslash\{k+1, k+2\}$ in $n$ subsets of size 2 . For every point $u$ 
of $\mathbb{H}_{n-1}$, let $\theta(u)$ denote the partition of $\{1,2, \ldots, 2 n+2\}$ obtained from $u$ by adding the subset $\{k+1, k+2\}$. Then $\theta$ defines an isomorphism between $\mathbb{H}_{n-1}$ and $\widetilde{M_{k+1}}$. We have

$$
\begin{gathered}
\theta^{-1}\left(x^{\prime}\right)=\{\{1,2\}, \ldots,\{k-2, k-1\},\{k, k+3\},\{k+4, k+5\}, \ldots,\{2 n+1,2 n+2\}\}, \\
\theta^{-1}(y)=\{\{2,3\}, \ldots,\{k-1, k\},\{k+3, k+4\}, \ldots,\{2 n+2,1\}\} .
\end{gathered}
$$

Now, define the following maxes:

$$
\begin{aligned}
M_{i}^{\prime} & :=M[i, i+1] \cap M_{k+1}, \quad i \in\{1, \ldots, k-1\} ; \\
M_{k}^{\prime} & :=M[k, k+3] \cap M_{k+1} ; \\
M_{i}^{\prime} & :=M[i+2, i+3] \cap M_{k+1}, \quad i \in\{k+1, \ldots, 2 n-1\} ; \\
M_{2 n}^{\prime} & :=M[1,2 n+2] \cap M_{k+1} .
\end{aligned}
$$

Then it is clear that $\left\{M_{1}^{\prime}, M_{2}^{\prime}, \ldots, M_{2 n}^{\prime}\right\}$ is the nice set of maxes of $\widetilde{M_{k+1}} \cong$ $\mathbb{H}_{n-1}$ induced by $\left(x^{\prime}, y\right)$. The $g_{n}(k, l)$ points of $\Delta_{l}(x) \cap \Delta_{n-l}(y) \cap M_{k+1}$ which are not contained in $M_{1} \cup M_{2} \cup \cdots \cup M_{k}$ are precisely the points of $\Delta_{l-1}\left(x^{\prime}\right) \cap$ $\Delta_{n-l}(y) \cap M_{k+1}$ which are not contained in $M_{1}^{\prime} \cup M_{2}^{\prime} \cup \cdots \cup M_{k-1}^{\prime}$. By Corollary $3.5(1)$, there are $g_{n}(k, l)=\sum_{i=k-1}^{n-1} g_{n-1}(i, l-1)$ such points.

By Lemmas 3.6, 3.7, 3.8, 3.9, 3.10 and Section 2.1, we have:

Corollary 3.11 For all $k, l \in\{0,1, \ldots, n\}$, we have

$g_{n}(k, l)=\left(\begin{array}{c}n-1-\left\lfloor\frac{k}{2}\right\rfloor \\ l-\left\lfloor\frac{k+1}{2}\right\rfloor\end{array}\right) \cdot\left(\begin{array}{c}n-\left\lfloor\frac{k+1}{2}\right\rfloor \\ l+\frac{(-1)^{k}-1}{2}\end{array}\right)-\left(\begin{array}{c}n-1-\left\lfloor\frac{k}{2}\right\rfloor \\ l-1-\left\lfloor\frac{k+1}{2}\right\rfloor\end{array}\right) \cdot\left(\begin{array}{c}n-\left\lfloor\frac{k+1}{2}\right\rfloor \\ l+\frac{(-1)^{k}+1}{2}\end{array}\right)$.

Proposition 3.12 For every $l \in\{0, \ldots, n\}$, we have $\left|\Delta_{l}(x) \cap \Delta_{n-l}(y)\right|=$ $\left(\begin{array}{c}n \\ l\end{array}\right)^{2}-\left(\begin{array}{c}n \\ l-1\end{array}\right) \cdot\left(\begin{array}{c}n \\ l+1\end{array}\right)$.

Proof. By Corollary 3.5(1), Lemma 3.8 and Corollary 3.11, we have

$$
\begin{aligned}
& \mid \Delta_{l}(x) \cap \Delta_{n-l}(y)=\sum_{i=0}^{n} g_{n}(i, l)=g_{n+1}(0, l) \\
= & \left(\begin{array}{c}
n \\
l
\end{array}\right) \cdot\left(\begin{array}{c}
n+1 \\
l
\end{array}\right)-\left(\begin{array}{c}
n \\
l-1
\end{array}\right) \cdot\left(\begin{array}{c}
n+1 \\
l+1
\end{array}\right) \\
= & \left(\begin{array}{l}
n \\
l
\end{array}\right) \cdot\left[\left(\begin{array}{c}
n+1 \\
l
\end{array}\right)-\left(\begin{array}{c}
n \\
l-1
\end{array}\right)\right]-\left(\begin{array}{c}
n \\
l-1
\end{array}\right) \cdot\left[\left(\begin{array}{c}
n+1 \\
l+1
\end{array}\right)-\left(\begin{array}{c}
n \\
l
\end{array}\right)\right] \\
= & \left(\begin{array}{l}
n \\
l
\end{array}\right)^{2}-\left(\begin{array}{c}
n \\
l-1
\end{array}\right) \cdot\left(\begin{array}{c}
n \\
l+1
\end{array}\right) .
\end{aligned}
$$


Remarks. (1) We have $|C(x, y)|=\sum_{l=0}^{n}\left(\begin{array}{c}n \\ l\end{array}\right)^{2}-\left(\begin{array}{c}n \\ l-1\end{array}\right) \cdot\left(\begin{array}{c}n \\ l+1\end{array}\right)=\sum_{l=0}^{n}\left(\begin{array}{c}n \\ l\end{array}\right)$. $\left(\begin{array}{c}n \\ n-l\end{array}\right)-\sum_{l=0}^{n}\left(\begin{array}{c}n \\ l-1\end{array}\right) \cdot\left(\begin{array}{c}n \\ n-l-1\end{array}\right)=\left(\begin{array}{c}2 n \\ n\end{array}\right)-\left(\begin{array}{c}2 n \\ n-2\end{array}\right)=\frac{1}{n+2}\left(\begin{array}{c}2 n+2 \\ n+1\end{array}\right)$. It was already mentioned above that $Y^{*}=C(x, y)$ and $\left|Y^{*}\right|=\frac{1}{n+2}\left(\begin{array}{c}2 n+2 \\ n+1\end{array}\right)$.

(2) Notice that the conclusion of Proposition 3.12 is also valid for the near polygon $\mathbb{H}_{1}$ (which is a line with three points).

\section{Proof of Theorem 1.2}

Theorem 1.2 trivially holds if $n=1$. So, we will suppose that $n \geq 2$.

Lemma 4.1 Suppose $e$ is the absolutely universal embedding of $\mathbb{H}_{n}$ into $\mathrm{PG}(W)$ and that $x$ and $y$ are two opposite points of $\mathbb{H}_{n}$. If $W_{i}, i \in\{0, \ldots, n\}$, is the subspace of $W$ for which $\mathrm{PG}\left(W_{i}\right)=\left\langle e\left(\Delta_{i}(x) \cap \Delta_{n-i}(y)\right)\right\rangle$, then

(1) $W=W_{0} \oplus W_{1} \oplus \cdots \oplus W_{n}$,

(2) $\operatorname{dim}\left(W_{i}\right)=\left(\begin{array}{c}n \\ i\end{array}\right)^{2}-\left(\begin{array}{c}n \\ i-1\end{array}\right) \cdot\left(\begin{array}{c}n \\ i+1\end{array}\right)$ for every $i \in\{0, \ldots, n\}$.

Proof. In Blokhuis and Brouwer [1], it as shown that $e(C(x, y))$ is an independent generating set of points of the projective space $\operatorname{PG}(W)$. The lemma then follows from Proposition 3.12.

Let $V$ be a $(2 n+2)$-dimensional vector space over $\mathbb{F}_{2}$ with basis $\left\{\bar{e}_{1}, \bar{e}_{2}, \ldots\right.$, $\left.\bar{e}_{2 n+2}\right\}$. Put $\bar{e}=\bar{e}_{1}+\bar{e}_{2}+\cdots+\bar{e}_{2 n+2}$. Let $V^{\prime}$ denote the subspace of $V$ consisting of all vectors of the form $\sum_{i=1}^{2 n+2} \lambda_{i} \bar{e}_{i}$ where $\lambda_{1}+\lambda_{2}+\cdots+\lambda_{2 n+2}=0$. If $\bar{x}=\sum_{i=1}^{2 n+2} \lambda_{i} \bar{e}_{i}$ and $\bar{y}=\sum_{i=1}^{2 n+2} \mu_{i} \bar{e}_{i}$ are two vectors of $V^{\prime}$, then we define $(\bar{x}, \bar{y})=\sum_{i=1}^{2 n+2} \lambda_{i} \mu_{i}$. Then $(\cdot, \cdot)$ is an alternating bilinear form on $V^{\prime}$ whose radical is equal to $\langle\bar{e}\rangle$. Let $D W(2 n-1,2)$ denote the point-line geometry whose points, respectively lines, are the $(n+1)$-dimensional, respectively $n$-dimensional, subspaces of $V^{\prime}$ which are totally isotropic with respect to $(\cdot, \cdot)$, with incidence being reverse containment. Then $D W(2 n-1,2)$ is a symplectic dual polar space of rank $n$. Let $A$ and $B$ denote the following mutually opposite points of $D W(2 n-1,2)$ :

$$
\begin{aligned}
& A=\left\langle\bar{e}, \bar{e}_{1}+\bar{e}_{2}, \bar{e}_{3}+\bar{e}_{4}, \ldots, \bar{e}_{2 n-1}+\bar{e}_{2 n}\right\rangle \\
& B=\left\langle\bar{e}, \bar{e}_{2}+\bar{e}_{3}, \bar{e}_{4}+\bar{e}_{5}, \ldots, \bar{e}_{2 n}+\bar{e}_{2 n+1}\right\rangle .
\end{aligned}
$$

We can write

$$
\bar{e} \wedge \bigwedge^{n} V^{\prime}=U_{0} \oplus U_{1} \oplus \cdots \oplus U_{n}
$$


where $U_{i}, i \in\{0, \ldots, n\}$, is the subspace of $\bigwedge^{n+1} V^{\prime}$ generated by all vectors of the form $\bar{e} \wedge \bar{a}_{1} \wedge \cdots \bar{a}_{n-i} \wedge \bar{b}_{n-i+1} \wedge \cdots \wedge \bar{b}_{n}$, where $\bar{a}_{1}, \ldots, \bar{a}_{n-i} \in A$ and $\bar{b}_{n-i+1}, \ldots, \bar{b}_{n} \in B$. For every point $p=\left\langle\bar{v}_{1}, \bar{v}_{2}, \ldots, \bar{v}_{n+1}\right\rangle$ of $D W(2 n-1,2)$, let $f(p)$ denote the vector $\bar{v}_{1} \wedge \bar{v}_{2} \wedge \cdots \wedge \bar{v}_{n+1}$ of $\bigwedge^{n+1} V^{\prime}$. The point $f(p)$ is independent from the generating set $\left\{\bar{v}_{1}, \bar{v}_{2}, \ldots, \bar{v}_{n+1}\right\}$ of $p$.

Suppose $p_{1}=\left\langle\bar{v}_{1}, \bar{v}_{2}, \ldots, \bar{v}_{n+1}\right\rangle$ is a point of $D W(2 n-1,2)$ at distance $i$ from $A$ and distance $n-i$ from $B$. Then $\left\langle\bar{v}_{1}, \bar{v}_{2}, \ldots, \bar{v}_{n+1}\right\rangle$ intersects $A$ in an $(n+1-i)$-dimensional subspace containing $\bar{e}$ and $B$ in an $(i+1)$-dimensional subspace containing $\bar{e}$. So, without loss of generality, we may suppose that $\bar{v}_{1}=\bar{e},\left\langle\bar{v}_{2}, \ldots, \bar{v}_{n+1-i}\right\rangle \subseteq A$ and $\left\langle\bar{v}_{n+2-i}, \ldots, \bar{v}_{n+1}\right\rangle \subseteq B$. It is then clear that

$$
f\left(p_{1}\right) \in U_{i} .
$$

Suppose $p_{2}=\left\langle\bar{v}_{1}, \bar{v}_{2}, \ldots, \bar{v}_{n+1}\right\rangle$ is a point of $D W(2 n-1,2)$ at distance at most $i$ from $A$. Then $\left\langle\bar{v}_{1}, \bar{v}_{2}, \ldots, \bar{v}_{n+1}\right\rangle$ intersects $A$ in a subspace of dimension at least $n+1-i$ which contains $\bar{e}$. So, without loss of generality, we may suppose that $\bar{v}_{1}=\bar{e}$ and $\left\langle\bar{v}_{2}, \ldots, \bar{v}_{n+1-i}\right\rangle \subseteq A$. It is then clear that

$$
f\left(p_{2}\right) \in U_{0} \oplus U_{1} \oplus \cdots \oplus U_{i}
$$

Similarly, as in the previous paragraph, one shows that if $p_{3}=\left\langle\bar{v}_{1}, \bar{v}_{2}, \ldots\right.$, $\left.\bar{v}_{n+1}\right\rangle$ is a point of $D W(2 n-1,2)$ at distance at most $i$ from $B$, then

$$
f\left(p_{3}\right) \in U_{n-i} \oplus U_{n+1-i} \oplus \cdots \oplus U_{n} .
$$

Now, suppose the points of $\mathbb{H}_{n}$ are the partitions of $\{1,2, \ldots, 2 n+2\}$ in $n+1$ subsets of size 2 . Let $x$ and $y$ be two opposite points of $\mathbb{H}_{n}$. Without loss of generality, we may suppose that $x=\{\{1,2\},\{3,4\}, \ldots,\{2 n+1,2 n+2\}\}$ and $y=\{\{2,3\},\{4,5\}, \ldots,\{2 n+2,1\}\}$. For every point $\left\{\left\{i_{1}, i_{2}\right\},\left\{i_{3}, i_{4}\right\}, \ldots\right.$, $\left.\left\{i_{2 n+1}, i_{2 n+2}\right\}\right\}$ of $\mathbb{H}_{n}$, we define $\epsilon(p)=\left\langle\bar{e}_{i_{1}}+\bar{e}_{i_{2}}, \bar{e}_{i_{3}}+\bar{e}_{i_{4}}, \ldots, \bar{e}_{i_{2 n+1}}+\bar{e}_{i_{2 n+2}}\right\rangle$. Then by Brouwer et al. [2, p. 356], $\epsilon$ is a full isometric embedding of $\mathbb{H}_{n}$ into $D W(2 n-1,2)$, i.e. $\epsilon$ is a map from the point set of $\mathbb{H}_{n}$ to the point set of $D W(2 n-1,2)$ which maps lines to lines and preserves the distances between points. Notice that $f \circ \epsilon$ is isomorphic to the absolutely universal embedding of $\mathbb{H}_{n}$. Also, $\epsilon(x)=A$ and $\epsilon(y)=B$. Theorem 1.2 is now a consequence of Lemma 4.1 and equations (1), (2), (3), (4).

\section{Acknowledgment}

At the moment of the writing of this paper, the author was a Postdoctoral Fellow of the Research Foundation - Flanders (Belgium). 


\section{References}

[1] A. Blokhuis and A. E. Brouwer. The universal embedding dimension of the near polygon on the 1-factors of a complete graph. Des. Codes Cryptogr. 17 (1999), 299-303.

[2] A. E. Brouwer, A. M. Cohen, J. I. Hall and H. A. Wilbrink. Near polygons and Fischer spaces. Geom. Dedicata 49 (1994), 349-368.

[3] A. E. Brouwer and H. A. Wilbrink. The structure of near polygons with quads. Geom. Dedicata 14 (1983), 145-176.

[4] B. De Bruyn. Near polygons. Frontiers in Mathematics. Birkhäuser Verlag, Basel, 2006.

[5] B. De Bruyn. A decomposition of the natural embedding spaces for the symplectic dual polar spaces. Linear Algebra Appl. 426 (2007), 462-477.

[6] B. De Bruyn. The structure of the spin-embeddings of dual polar spaces and related geometries. European J. Combin. 29 (2008), 1242-1256.

[7] B. De Bruyn. On the Grassmann-embeddings of the hermitian dual polar spaces. Linear Multilinear Algebra 56 (2008), 665-677.

[8] B. De Bruyn. Some subspaces of the $k$-th exterior power of a symplectic vector space. Linear Algebra Appl. 430 (2009), 3095-3104.

[9] J. H. van Lint. Combinatorial Theory Seminar, Eindhoven University of Technology. Lecture Notes in Mathematics 382. Springer-Verlag, BerlinNew York, 1974.

[10] S. E. Payne and J. A. Thas. Finite generalized quadrangles. Second edition. EMS Series of Lectures in Mathematics. European Mathematical Society (EMS), Zürich, 2009.

[11] M. A. Ronan. Embeddings and hyperplanes of discrete geometries. European J. Combin. 8 (1987), 179-185.

[12] E. E. Shult and A. Yanushka. Near $n$-gons and line systems. Geom. Dedicata 9 (1980), 1-72. 